\title{
Angio-seal infection: An uncommon but serious and alarming complication of vascular closure devices
}

\author{
Pradeep Kumar Mada ${ }^{*}$, Liam Morris ${ }^{2}$, Pavan Katikaneni ${ }^{2}$ and Andrew Stevenson Joel Chandranesan ${ }^{1}$ \\ ${ }^{1}$ Department of Infectious diseases, Louisiana State University Health Sciences Center Shreveport, USA \\ ${ }^{2}$ Department of Interventional Cardiology, Louisiana State University Health Sciences Center Shreveport, USA
}

\section{Background}

Femoral arterial access is the method that is widely used to gain vascular access for performing diagnostic and therapeutic interventions such as coronary angiography and percutaneous coronary intervention. In the past manual compression was used to achieve hemostasis which over the past two decades has been largely replaced by vascular closure devices (VCD). Different types of vascular closure devices such as collagen based plug devices (Angio-seal), polyglycolic acid based plug devices (Mynx Ace, Exo seal), clip closure devices (Starclose), and percutaneous suture closure devices (Perclose) are commonly used for achieving hemostasis [1]. Several factors such as minimal patient discomfort, earlier ambulation, and reduction in the personnel required when compared to manual compression resulted in an increased use of vascular closure devices [2]. Complications associated with the use of these devices include bleeding, hematoma, aneurysm, infection, arterio-venous fistula, allergic reaction, and foreign body reaction [3]. A study performed to estimate the rate of complications revealed that $2 \%$ of the patients develop the above-mentioned complications and infections account for about $0.3 \%$ of these complications [4].

While the rate of noninfectious adverse events is almost similar in manual compression and the use of these devices the rate of infectious complications is slightly higher with the use of vascular closure devices [5,6]. Age between 40-79 years, Diabetes mellitus and obesity have been identified as the possible risk factors for the occurrence of infections following the use of such devices [7]. Staphylococcal aureus accounts to $75 \%$ of infections in these cases, followed by gram negative rods in $13 \%$ and coagulase negative staphylococcus in $5 \%$ of the cases [3]. Here in we describe a case of 52-year-old female who underwent percutaneous coronary intervention followed by the use of Angio-seal, a vascular closure device and later developed wound infection caused by streptococcus agalactiae.

\section{Case summary}

A 52-year-old Asian female was evaluated for Canadian cardiovascular society class 3 angina which was refractory to medical therapy. The patient had a past medical history of Diabetes Mellitus, Hypertension, Hyperlipidemia, Coronary Artery Disease status post percutaneous coronary intervention (PCI) in 2005 to proximal left anterior descending artery (LAD) and right coronary artery (RCA), PCI in 2014 to right coronary artery. She was referred to our cardiology department for coronary angiography for further evaluation. Under aseptic conditions, the right femoral artery was cannulated and a vascular sheath was placed. Selective coronary angiography was then performed of the left and right coronary systems in multiple projections using diagnostic catheters. Findings revealed significant
LAD disease (Mid and distal left anterior descending stents are patent with mild intra stent stenosis. Just distal to the edge of the distal LAD stent there is diffuse $70 \%$ disease. This is followed by a $90 \%$ stenosis in the apical LAD), and she underwent successful percutaneous coronary intervention to her LAD.

The right femoral arterial sheath was subsequently discontinued, and a Vascular Closure Device (Angio - Seal) was used to achieve hemostasis. Groin Check four hours later revealed a scant ooze, with no signs of active bleeding or hematoma formation. Neurological exam was intact and distal pulses were palpable. The patient tolerated the procedure well and was returned to the recovery room in stable condition. She was discharged in stable condition on the next day. Two days after her discharge, she developed a fever of 103 Fahrenheit associated with chills and myalgias. She denied pain or discharge from the puncture site, shortness of breath, cough, abdominal pain, vomiting, diarrhea, or dysuria. On admission, her signs were as follows: temperature 102 Fahrenheit, pulse rate 100 per minute, respiratory rate 18 per minute and blood pressure $110 / 55$ millimeter of mercury. Physical examination did not reveal any abnormalities including her groin site which was clean without any fluctuance, erythema or drainage. Bed side ultrasound of right groin site did not reveal any fluid collection. The patient was started on a combination of broad spectrum antibiotics vancomycin and piperacillin/tazobactam. Blood cultures came back positive for Streptococcus agalactiae. Vascular surgery and infectious diseases consultations were then placed. The next day, the wound site expressed frank pus (Figure 1), after which the patient was rushed to the operating room and wound exploration revealed $2 \times 3$ centimeters pocket of pus below the right groin puncture site and a hematoma in the right groin. Surgical debridement was performed and wound closure was performed with a Jackson-pott drain in place. The tissue from the site of debridement was cultured and it revealed Streptococcus agalactiae and Enterobacter aerogenes. Enterobacter was sensitive to quinolones and intermediately sensitive to Piperacillin/ Tazobactam. Based on culture and sensitivity report, her antibiotics were switched to ceftriaxone and ciprofloxacin. The patient continued to have intermittent low-grade fevers post debridement. She was then suspected to have a seroma on physical examination and removal of a suture revealed 2-3 milliliters of dark turbid fluid. A Transesophageal echocardiogram was performed to evaluate for Infective Endocarditis,

Correspondence to: Pradeep Kumar Mada, Department of Infectious diseases, Louisiana State University Health Sciences Center Shreveport, USA, Tel: 318469-1416, E-mail: pmada@lsuhsc.edu

Received: June 08, 2017; Accepted: June 23, 2017; Published: June 26, 2017 


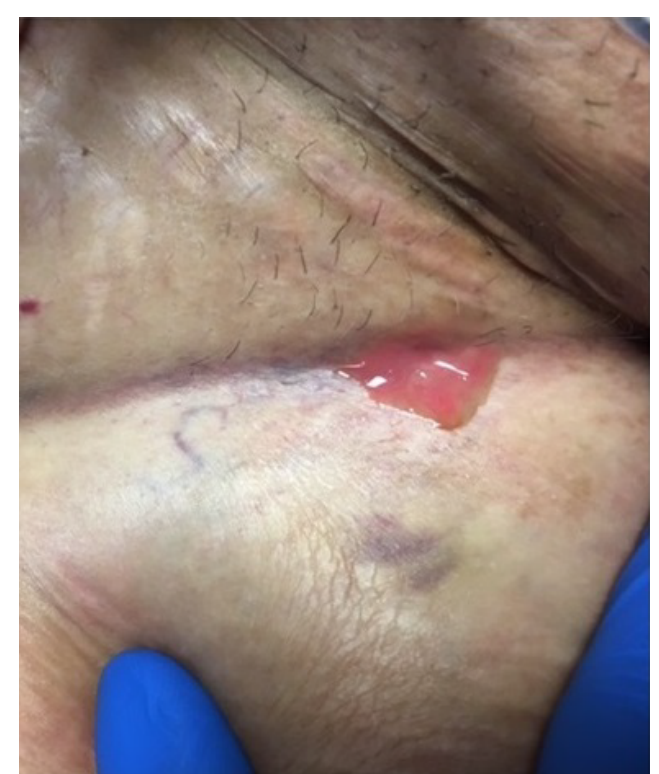

Figure 1. Right groin puncture site expressing frank pus.

it was however, negative for any vegetation or thrombus. Computed Tomography scan of abdomen and pelvis revealed postsurgical changes in the right inguinal area from prior right common femoral access with soft tissue inflammation and a small hematoma. Repeat surgical debridement was performed with placement of Negativepressure wound therapy 10 days after the initial debridement was done. Her fever resolved and repeat blood cultures showed no growth. She was continued on ceftriaxone and ciprofloxacin for two weeks from the last wound debridement. She was discharged to home in stable condition. We saw her in clinic after one month. She was doing well with no complaints. Her right groin wound healed well with no tenderness on exam and no signs of infection.

\section{Discussion}

Angio-seal closure device used in this case creates a mechanical seal by sandwiching the arteriotomy site between a bio absorbable anchor and a collagen sponge. The collagen sponge and the anchor dissolve gradually over a period of 60-90 days [8]. Infection of the wound site following the use of Angio-seal is extremely rare but is a serious complication. Among the risk factors implicated in the causation of infections by Vascular Closure Device (VCD), our patient had diabetes and was in the aforementioned age range. Presence of a foreign body or formation of hematoma have been described as the possible sources of infection in previous cases. The patient in this case did not have either of these contributing factors and the source of infection remains unclear. Furthermore, common causative agent in these cases is staphylococcal aureus but in case of the current patient two different organisms streptococcus agalactiae and Enterobacter aerogenes were implicated in the causation of the infection. Infection with these organisms were not reported in previous case reports that described wound infections associated with vascular closure devices.

As per the current guidelines routine antimicrobial prophylaxis is not recommended after the use of VCD. There exists limited information on the possible organisms that could be involved in the causation of wound infections associated with vascular closure device. However, future research might provide a better understanding of all possible risk factors and revised guidelines with a more cautious approach would be beneficial in reducing the recurrence of such infections.

\section{References}

1. Malekpour F, Castillo R (2015) Infection: A perilous complication of a closure device. Vascular Disease Management 12: E145-E151.

2. Schwartz BG, Burstein S, Economides C, Kloner RA, Shavelle D, Mayeda, G (2011) Review of vascular closure devices. Cath Lab Digest 19: 1,10-20.

3. Doriaswamy VA, SUgumaran R, Kern KB (2013) Femoral artery closure deviceschallenges of infection. Journal of Cardiology and Vascular Medicine 2: 1-4.

4. Smilowitz NR, Kirtane AJ, Guiry M, Gray WA, Dolcimascolo P, et al. (2012) Practices and complications of vascular closure devices and manual compression in patients undergoing elective transfemoral coronary procedures. Am J Cardiol 110: 177-182. [Crossref]

5. Applegate RJ, Sacrinty MT, Kutcher MA, Baki TT, Gandhi SK, et al. (2006) Propensity score analysis of vascular complications after diagnostic cardiac catheterization and percutaneous coronary intervention 1998-2003. Catheter Cardiovasc Interv 67: 556562. [Crossref]

6. Ramsdale DR, Aziz S, Newall N, Palmer N, Jackson M (2004) Bacteremia following complex percutaneous coronary intervention. J Invasive Cardiol 16: 632-634. [Crossref]

7. Franco J, Motaganahalli R, Habeeb M, Wittgen C, Peterson G (2009) Risk factors for infectious complications with angio-seal percutaneous vascular closure devices. Vascular 17: 218-221. [Crossref]

8. Nash JE, Evans DG (1999) The Angio-Seal hemostatic puncture closure device. Concept and experimental results. Herz 24: 597-606. [Crossref]

Copyright: (C2017 Mada PK. This is an open-access article distributed under the terms of the Creative Commons Attribution License, which permits unrestricted use, distribution, and reproduction in any medium, provided the original author and source are credited. 